\title{
Discovery of a Tight Brown Dwarf Companion to the Low Mass Star LHS 2397a
}

\author{
Melanie Freed, Laird M. Close, \& Nick Siegler \\ Steward Observatory, University of Arizona, 933 North Cherry Avenue, \\ Tucson AZ, USA 85721-0065; mfreed@as.arizona.edu
}

\begin{abstract}
Using the adaptive optics system, Hōkūpa'a, at GeminiNorth, we have directly imaged a companion around the UKIRT faint standard M8 star, LHS 2397a (FS 129) at a separation of 2.96 AU. NearInfrared photometry obtained on the companion has shown it to be an L7.5 brown dwarf and confirmed the spectral type of the primary to be an M8. We also derive a substellar mass of the companion of $0.068 M_{\odot}$, although masses in the range $(0.061-0.069)$ are possible, and the primary mass as $0.090 M_{\odot}(0.089-0.094)$. Reanalysis of archival imaging from HST has confirmed the secondary as a common proper motion object. This binary represents the first clear example of a brown dwarf companion within $4 \mathrm{AU}$ of a low mass star, and should be the first L7.5 to have a dynamical mass. As part of a larger survey of M8-M9 stars, this object may indicate that there is no "brown dwarf desert" around low mass primaries.
\end{abstract}

\section{Introduction}

Radial velocity surveys have observed a lack of brown dwarf companions to Sunlike stars, the "brown dwarf desert". Marcy \& Butler (2000) estimate that less than $0.5 \%$ of Sun-like stars have brown dwarf companions within 3AU.

The question still remains as to whether lower mass primaries also exhibit a "brown dwarf desert". Here we present the discovery of a brown dwarf companion orbiting within $4 \mathrm{AU}$ of the nearby UKIRT faint standard M8 star, LHS 2397a (FS 129). This is the first clear example of a brown dwarf companion to a low mass star (later than M2) within 4 AU and may suggest that no "brown dwarf desert" exists for low mass primaries. Given the small separation of this system, dynamical masses can be determined in a relatively short period of time $(\sim 5 \mathrm{yr})$, which will help calibrate mass-luminosity isochrone models and the bottom of the main sequence. Please see Freed et al. (2002) for a more thorough treatment of this data.

\section{Observations, Photometry, \& Spectral Type}

Using the adaptive optics system at Gemini-North, we acquired near-diffraction limited images of LHS 2397a in JHK' bands on 2002 February 7, UT. We also 


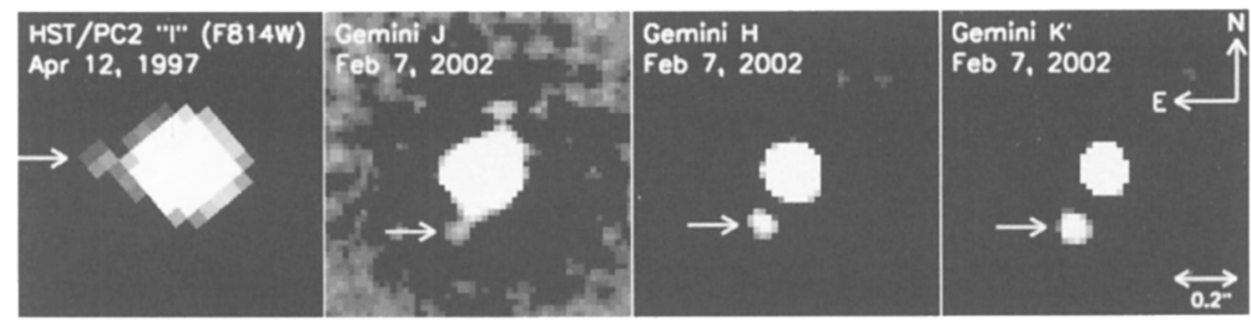

Figure 1. Images of LHS 2397a taken in all four bandpasses.

obtained two $\mathrm{F} 814 \mathrm{~W}(\mathrm{CWL}=0.82 \mu \mathrm{m})$ images taken on 1997 April 12, UT from the HST public archival database (Kirkpatrick \& Henry 1997).

Differential magnitudes and their errors were determined for each of the four bands using the DAOPHOT (Stetson 1987) package in IRAF and used to derive individual absolute magnitudes. Positions were determined as well for the F814W and K' images.

To determine the spectral types of the two components, we compared our calculated absolute magnitudes, in all four bands, with the absolute magnitude vs. spectral type relationships derived by (Dahn et al. 2002) and Ks magnitudes from 2MASS. For every observed bandpass, we confirm that the primary is an M8 and the secondary is consistent with an L7.5. Therefore, we conclude that the companion is a brown dwarf.

\section{Space and Orbital Motions \& Mass}

Our AO observations are nearly 5 years after the HST images, which allows us to verify that the binary components are common proper motion as well as observe significant orbital motion. We calculate a probably that the secondary is a background object to be a negligible $10^{-18}$. As a result, taking into account orbital motion, these two objects form a common proper motion pair and are, therefore, physically associated. We denote the two components as LHS 2397aA and LHS 2397aB.

Given the observed orbital motion, we estimate the period of the orbit to be $25 \mathrm{yr}$, which is not inconsistent with a substellar object orbiting LHS 2397a. While two epochs of an orbit are insufficient to give a dynamical mass, we should be able to do so in about another 5 years.

Since the complete space motions of LHS 2397a are known, we use kinematic arguments to estimate the age range to be 2.0-12.0 Gyr with a best guess age of 7.2 Gyr. Our derived absolute Ks magnitudes, in conjunction with this age range, for LHS 2397aA and LHS 2397aB were compared to Chabrier et al. (2000)'s DUSTY Ks models to derive a primary mass of $0.090 M_{\odot}(0.089-0.094)$ and a secondary mass of $0.068 M_{\odot}(0.061-0.069)$. Given these masses and assuming the semi-major axis of the orbit to be $3.86 \mathrm{AU}$, we derive a period of the system of $19 \mathrm{yr}$, which is consistent with the previously derived characteristic period.

For the full range of $M_{K s}$ and age values, we find that the primary is a star and the secondary is unambiguously a brown dwarf. 
Acknowledgments. We are indebted to I. Baraffe \& her collaborators for kindly providing us with custom DUSTY models. We are also grateful to E. Mamajek, J.Liebert, S. Shaklan, S.Unwin, P.Butler, D.Queloz, and V.Caloi for various helpful information. We acknowledge support from the AFOSR under F49620-01-1-0383 and NASA Origins of Solar Systems grant NAG5-12086. These results were based on observations obtained at the Gemini Observatory. Based on observations made with the NASA/ESA Hubble Space Telescope, obtained from the data archive at the Space Telescope Science Institute. This publication makes use of data products from the Two Micron All Sky Survey.

\section{References}

Chabrier, G., Baraffe, I., Allard, F., \& Hauschildt, P. 2000, ApJ, 542, 464

Dahn, C.C., et al. 2002, AJ, in press (astro-ph/0205050)

Freed, M., Close, L.M., \& Siegler, N. 2002, submitted ApJ

Kirkpatrick, J.D., \& Henry, T.J. 1997, HST Proposal 6345

Marcy, G.W., \& Butler, R.P. 2000, PASP, 112, 137

Stetson, P.B. 1987, PASP, 99, 191 


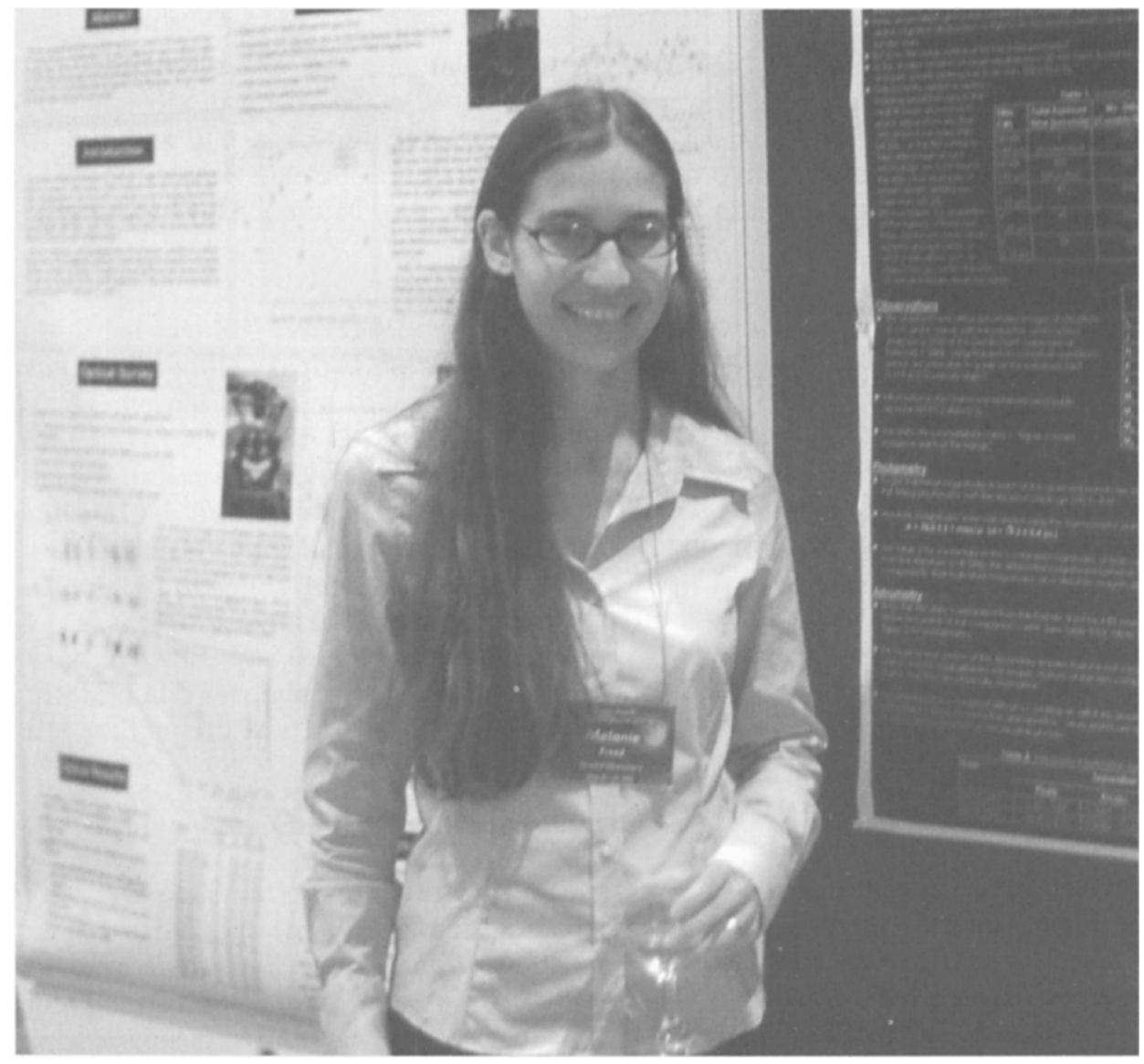

Melanie Freed 